\title{
Programming Research on Working Performance Tables of All-Terrain Cranes
}

\author{
Yixiao Qin*, Qingqing Yang, Tongzun Wang \\ College of Mechanical Engineering, Taiyuan University of Science and Technology, Taiyuan, China
}

\author{
Email address: \\ qyx819@163.com (Yixiao Qin), 317426237@qq.com (Qingqing Yang) \\ ${ }^{*}$ Corresponding author
}

\section{To cite this article:}

Yixiao Qin, Qingqing Yang, Tongzun Wang. Programming Research on Working Performance Tables of All-Terrain Cranes. International Journal of Science and Qualitative Analysis. Vol. 3, No. 1, 2017, pp. 1-9. doi: 10.11648/j.ijsqa.20170301.11

Received: December 30, 2016; Accepted: January 14, 2017; Published: February 13, 2017

\begin{abstract}
An all-terrain crane is a lifting machine that can simultaneously complete vertical lifting for cargoes and horizontal movement of machinery. There are boom structure combinations up to more than 10 thousand kinds when an all-terrain crane works, so it is very difficult to calculate the performance of the arm frame considering all working conditions and the stability of the whole machine, thus the workload of making performance tables is enormous and it is very difficult in actual operation. Therefore, in order that making crane performance tables becomes more reliable and efficient, applying object-oriented programming techniques to making crane performance tables and automatic document output of performance tables are studied in this paper, which can greatly improve the efficiency of development of new products for all-terrain cranes.
\end{abstract}

Keywords: All-Terrain Cranes, Mechanics Analysis, Performance Tables, Programming Realization

\section{Introduction}

Larger all-terrain cranes are increasing with the development of oil industry, chemical industry and wind power industry in recent years, at the same time completing steadily vertical lifting for cargoes and realizing safely horizontal movement of machinery put forward to higher requirements to working performance of all-terrain cranes. Therefore the performance of the arm frame considering all working conditions and the stability of the whole machine has been a hot research topic in industry for a long time, but working conditions of arm frame for all-terrain cranes are numerous, so it is very difficult to calculate the performance of arm frame. According to the above problem, the related researches have been done before. This paper aimed to carry out a research on its structural performance based on the method of theoretical calculation and finite element analysis because of the huge lifting weight and complex structure of large-tonnage gantry cranes and in order to effectively design and review it, which also verified that the method of finite element is an effective way to find a real dangerous cross-section [1]. The parametric model could be used for various vice arm cranes design cases, and accessing to the rules from any design procedure was fully automated by using a systematic approach of parametric modeling in this paper [2]. One of the important factors in some achievements was the weight reduction of the boom without sacrificing their lifting capacity, to this end, structural analyses of the boom has been performed using finite-element simulations [3]. The paper was a review of enhanced concept of computer aided structural analysis software application in FEM modeling of spatial structural systems of bridges, buildings, industrial facilities, machine devices, and evaluated procedures of real structural performances [4]. Now excel VBA was used for batch calculation in local singularity analysis [5]. Research on object oriented programming was carried on [6]. The article introduced these functions: the exploit environment and syntax of VBA technology, the new general control language of windows practical software, and combining the exam paper analysis in the exam, subjecting the design proposal to automatic producing the exam paper analysis using VBA technology [7]. Frame structures for lift crane machinery were introduced [8]. Based on the geometric nonlinearity beam theory, these all-terrain cranes and their components were 
simplified to the beam unit, Matlab programming was used to calculate the boom strength of all-terrain cranes [9]. A weak form Galerkin finite element model for the nonlinear quasi-static and fully transient analysis of initially straight viscoelastic beams was developed using the kinematic assumptions of the third-order Reddy beam theory [10]. When an all-terrain crane is in operation, arm frame structure indicates big deformation state. To figure out non-linear strength solution and buckling solution of certain actual telescopic arm frame, beam element and refined net were used for modeling to get accurate result and the convergence solution, which can provide guidance for product design [11]. A nonlinear structure analysis by the finite element analysis was carried out to investigate the failure reason of an all-terrain crane telescopic boom, an overall simplified model consisting of telescopic boom and luffing vice arm was established by beam element, and analyzed using geometric nonlinear static method. The connection of load, boom buckling failure and stress wrinkle were studied by using nonlinear static method and explicit dynamic method [12]. Larger all-terrain design development process is a very complex process. For crane structure design, considering not only the strength of the performance of the structure, stiffness performance and stability is needed, and to consider the luffing hydraulic cylinder, super lifting rope and other structures is also necessary, so the finite element method is adopted in calculation of crane performance in this paper. And the previous traditional way of making performance tables is to select the typical dangerous conditions to calculate the lifting capacity of these working conditions, which can only reflect the typical working conditions, but not fully reflect the working safety of cranes when making the performance tables. Due to diversification of arm frame structure in the form of large-tonnage cranes and tens of thousands of working conditions, it is very difficult to calculate and make the performance tables in the actual operation when considering complicated performance calculation for all working conditions. Therefore, applying object-oriented programming techniques to making crane performance tables and automatic document output of performance tables are studied in this paper, which also greatly improves the efficiency of development of new products for all-terrain cranes, and it has also a certain significance, in the meantime, can shorten the product development cycle.

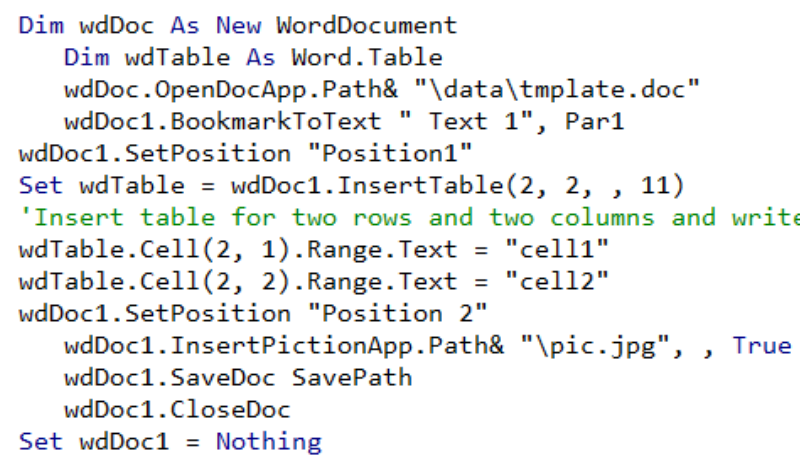

\section{Programming Implementation of Nonlinear Analysis in any Working Condition on an All-Terrain Crane Boom Structure}

\subsection{Program Development on Office Automation of Oriented Engineering}

Office automation technology based on Microsoft Office and VBA has been widely used in the template of a variety of reports and documents. As a tool language of Microsoft Corp, VBA is specifically designed to provide office program development, VBA is a standard macro language that can be applied widely and is easy to use, also realizing data interaction of office program and programming language like VB, VC. Because commonalities between VB language and VBA language, using VB language is more simple and efficient. Common office automation technology that is based on WORD and VBA technology has some methods like outputting document by directly using VBA programming and replacing parameters with bookmarks etc. The former method makes the contents of the output into inside of procedure, thus the output contents are easy to be controlled, which is suitable for various document formats and less document content; The latter method is to make the document into a generic template and the output contents are made into a bookmark, on output, it can find the specified bookmarks for replacement by using VBA, which is suitable for fixed relatively document format. Because the format of the structural design calculation reports is relatively fixed, this paper is based on the latter approach, the ANSYS analysis results and final results are outputted to calculation reports in WORD document formats or performance tables of all-terrain cranes by using VB programming, which is shown in figure 1.

In order to output more efficiently all-terrain crane performance tables by using VBA language, a commonly used VBA statements are encapsulated by making full use of VB 6.0 class programming in this paper, WorkDoc class module is written, simplifying output statement to make office automation programming is far more efficient, more quickly. The major contents about WorkDoc class modules are shown in figure 2 .

After defining WorkDoc class modules, while programming we only need to carry out a simple statement, it is ok that the result data are outputted to the word document, which is as follows.

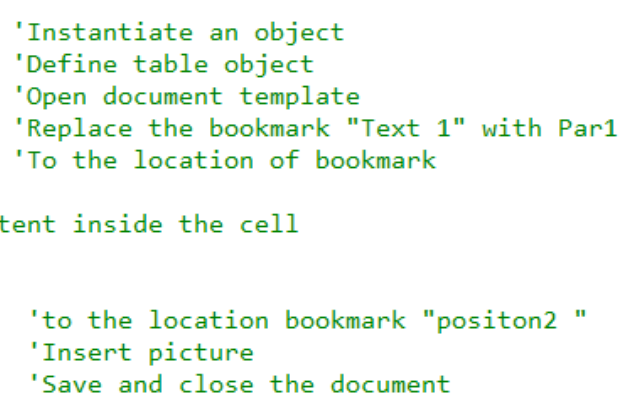

Figure 1. Codes of generating words. 


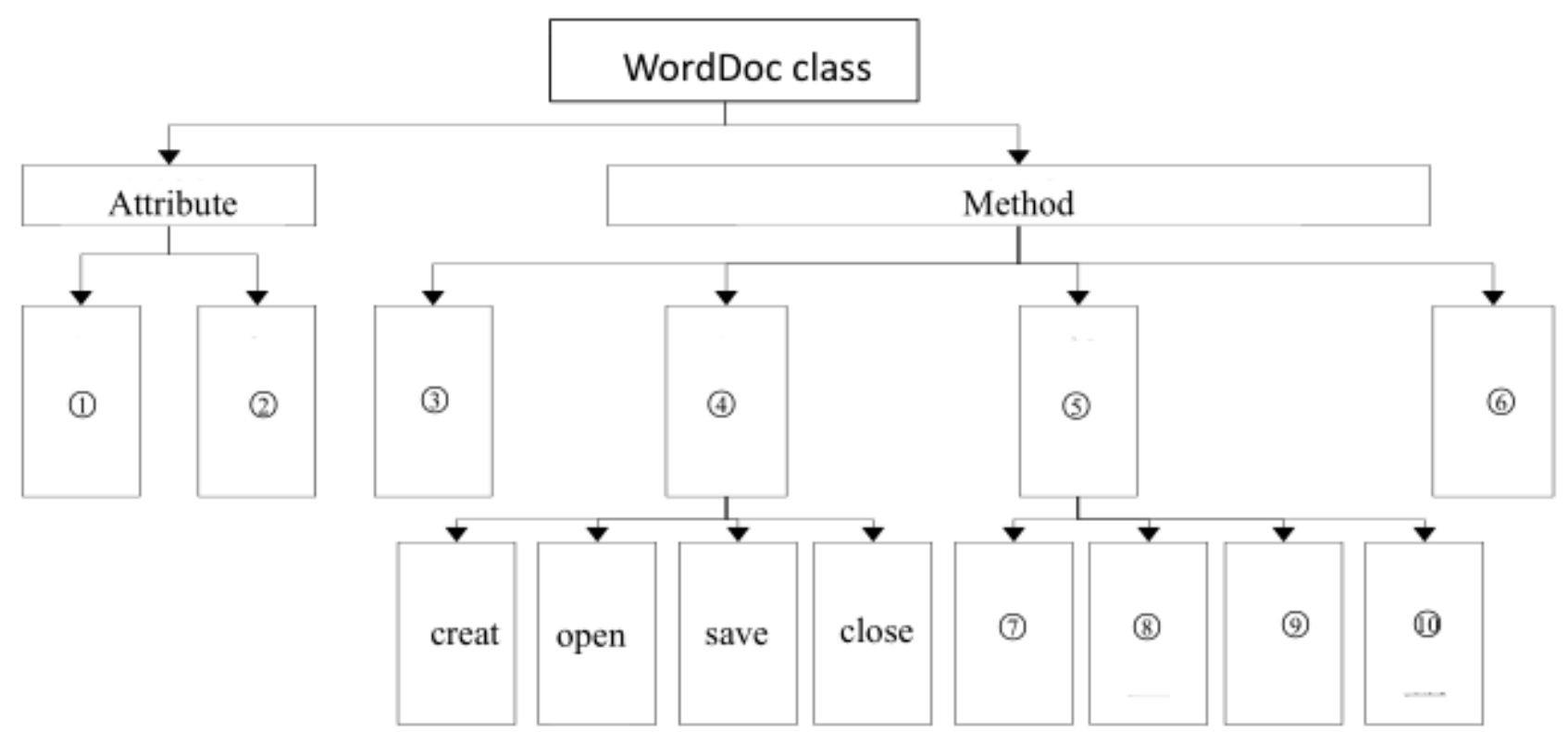

Figure 2. WordDoc class module.

(1) Paragraph attributes (2)Font properties (3) Initialization of class (4) Document operation (5)bookmarks operation (6)Destruction of class (7) Paragraph bookmarks (8) Text bookmarks (9) Picture bookmarks (10 Tables bookmarks

When the performance tables of all-terrain cranes are made, firstly, the analysis of the arm frame structure is carried out, which includes boom structure modeling, finite element analysis and analysis result determination, etc.

\subsection{Components of Boom Structure}

Based on the parametric design of object-oriented programming technology, various parts forms of arm frame structure are more fixed in this paper. A "bookmark" need to be formed for every part parameters of boom. The result data of boom structure though the finite element analysis is replaced by using VB programming. Various parameters of variable diameter section are shown in figure 3, which reappeared as a bookmark in the output document.

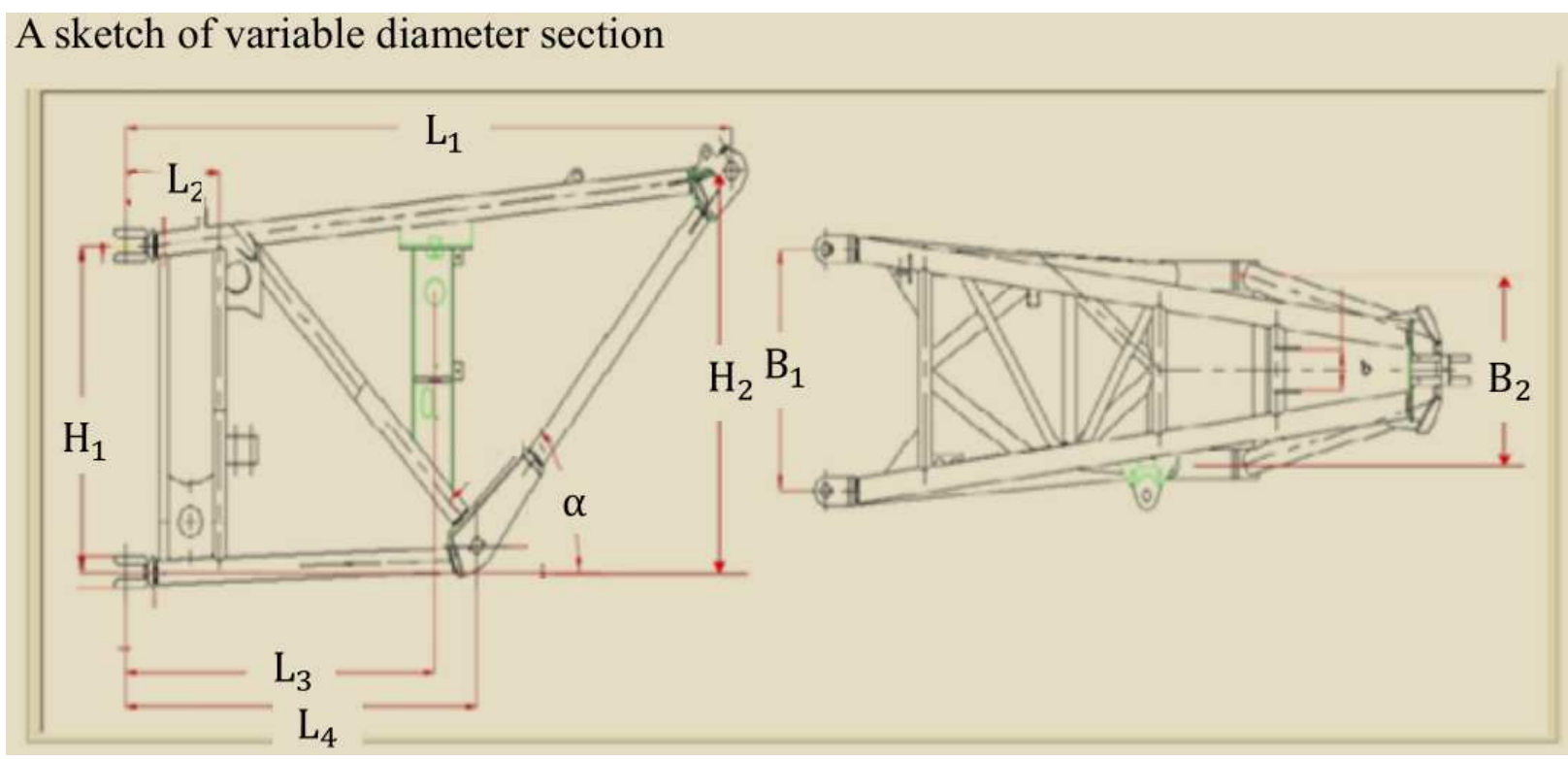

Figure 3. Bookmarks of boom structural parameters.

\subsection{Structural Calculation Working Condition Parameters}

Working condition contents of calculating the performance tables include mainly the combination form of arm frame structures while an all-terrain crane works, the load, the lifting rope ratio and so on, in this paper, the parameters of the working conditions are located mainly through the bookmark, results are outputted at the specified location and the parameters are shown in figure 4. 


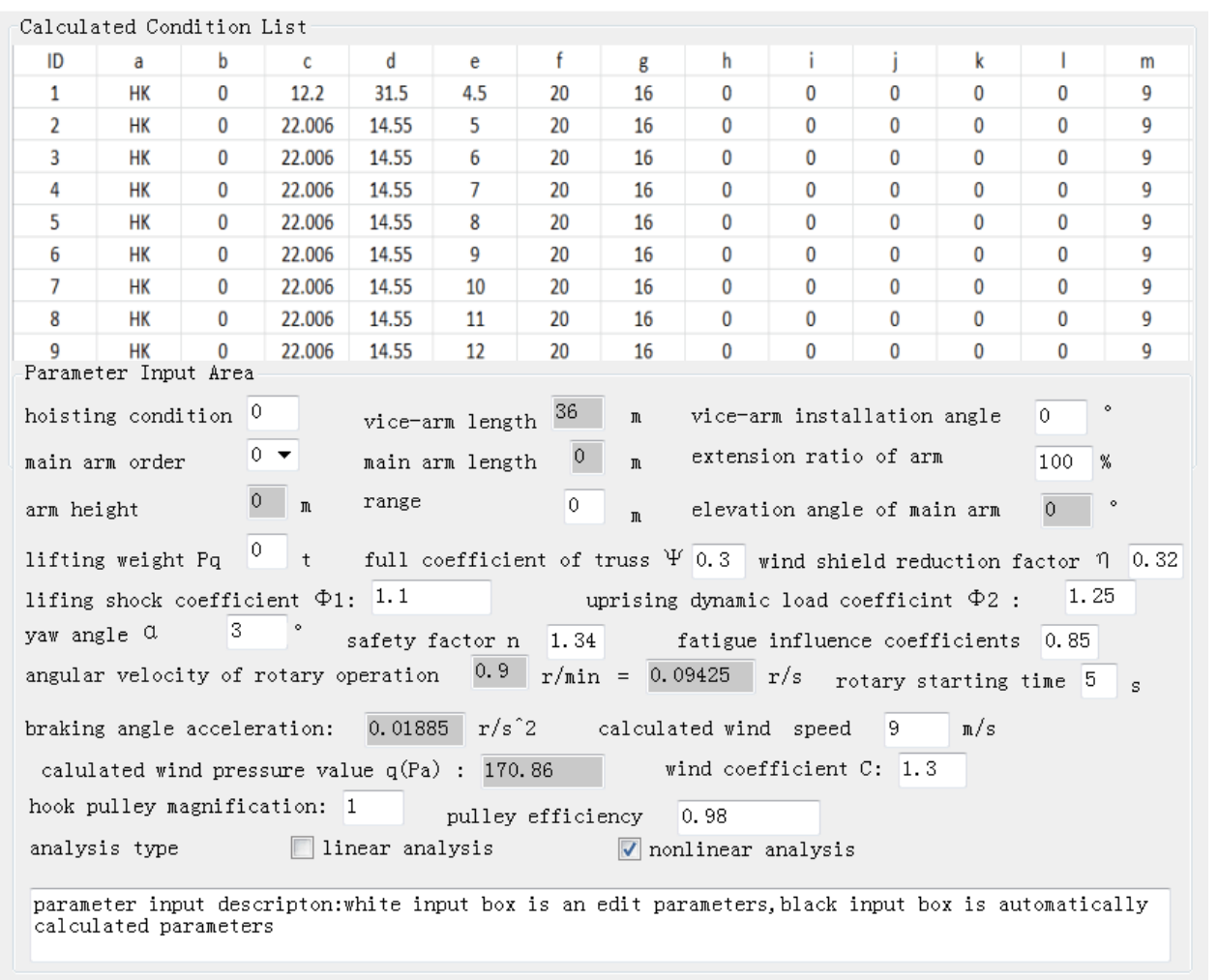

Figure 4. Working condition parameters.

a.vice-arm working condition; b.vice-arm installation angle; c.vice-arm length; d.main arm length; e.range; f.lifting weight; magnification; h.the first section ratio; i.the second section ratio; j.the third section ratio; $k$.the fourth section ratio; 1 .the fifth section ratio; m.wind speeds

\subsection{Finite Element Analysis of Boom Structure}

All-terrain crane structure is composed of two parts: the upper part of the car and the lower part of the car. The arm frame is mainly composed of telescopic main boom, derricking cylinder, super-lift system, truss vice arm, etc. There are a variety of forms formed by different combinations in the boom system of all-terrain cranes, common combination forms are shown in figure 5, including main arm, main arm and super-lift system, main arm and fixed vice arm, main arm and fixed vice arm and super-lift system, main arm and tower arms, main arm and tower arms and super-lift system.

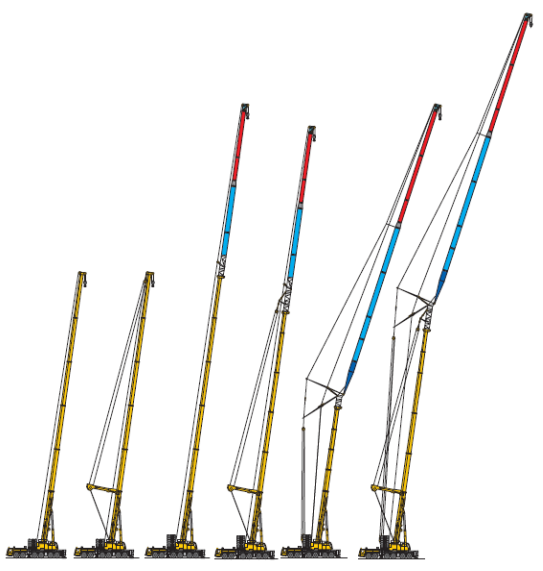

Figure 5. Common combinations of arm frame of an all-terrain crane.
In this paper, finite element analysis of arm frame structures is introduced about simplification of finite element model of boom structure, unit type and connection form each part of the structure adopted, load position and manner, the picture of the analysis results and list display, etc. These contents above are stored in the database, it is ok that the corresponding data results or graphic results are extracted and outputted when the corresponding parts are outputted.

In the determination of the analysis results, this paper mainly introduces the judgment criterion about the design of arm frame structure and determination of finite element analysis results. The enlarged image of the analysis result in the dangerous part is given, which is conducive to those checked problem of the structure and provides basis for further improvement of design. By defining bookmark of the parameters in the calculation reports, the finite element analysis results of arm frame structures can be easily outputted to word documents in the specified format, which is regard as the support basis for performance tables.

\section{Crane Performance Tables Programming}

Crane performance tables of all-terrain cranes include the structure size parameters and performance parameters of an crane, which are important reference for users to buy a crane and are also operations manuals that guides the user to carry out the actual operation, it plays a crucial role in the sales and 
use of all-terrain cranes, and is also important for accident analysis. The traditional calculation method about crane lifting performance tables is based on empirical formula to calculate, safety factor used in the calculation process is affected greatly by subjective factors, the accuracy of the calculation results cannot be guaranteed. In view of the importance of performance calculation, the finite element method is used for rapid modeling and analysis, so that it can optimize the performance calculation method and enhance the safety and reliability of the structure.

\subsection{The Performance Calculation of All-Terrain Cranes}

Main performance parameters of all-terrain cranes include the weight, amplitude, lifting height, magnification, and arm frame combinations and so on, as shown in figure 6. Main work of performance calculation is to calculate load under a certain length and amplitude. The traditional calculation method is to carry on strength checking calculation for the dangerous condition, which cannot fully reflect the crane's lifting status. Adopting finite element method to calculate a crane performance not only considers the strength and rigidity of the structure, but also considers inter force of luffing hydraulic cylinder, super lifting rope force, the tipping steadiness of the entire crane, leg force, rotary supporting force, etc. Through the comprehensive analysis of many aspects, the final performance is to take the minimum value of various controls as working performance of the corresponding conditions.

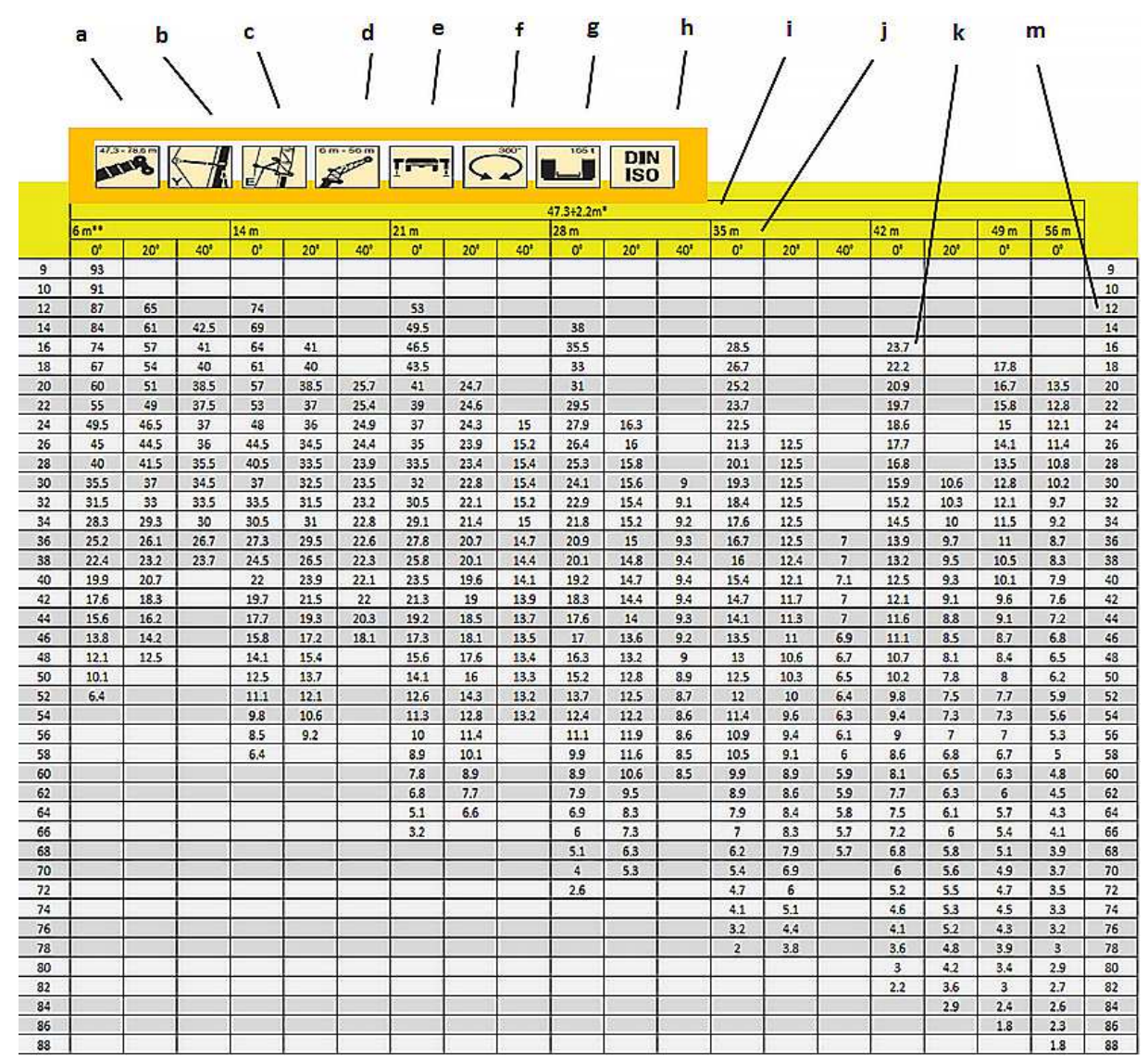

Figure 6. Lifting performance tables style of an al-terrain crane.

a - Telescope Boom Range b - Super Lifting Spars c — Luffing Jib Range d- Fixed Jib Range e- Body Focus Counterweight f — Working Range g — Body Counterweight $\mathrm{h}$ — German and international standards $\mathrm{i}$ — Telescope Boom Length $\mathrm{j}$ — Luffing Jib Length $\mathrm{k}$ — Rated Lifting Capacity $\mathrm{m}$ — Working Radius

Lifting performance calculation of all terrain cranes is regarded as the reverse process of structure design calculation, calculation verification of structure design refers to checking arm structure strength, stiffness and stability to meet the design requirements in a given load, calculation of lifting performance is carried on under the certain mechanism form, and by adjusting constantly sizes of these loads until the performance analysis of the structure in line with the design requirements, the latter is an constantly iterative solution process, we can use a certain optimization algorithm to find the optimal solution which matches the design, iteration is carried out by using the dichotomy in this paper, in general, the iteration is carried out for about 10 times for each working condition. It is ok to find the optimal solution to meet the requirements. The specific approach is firstly to establish the model of arm frame structure, which is analyzed under a given initial starting weight load. Sizes of these loads are adjusted constantly through judging the performance of the analysis under given control conditions to carry on Iteration, until all the control conditions are satisfied, in this paper, we use VB 6.0 to write the program flow chart as shown in figure 7 . 


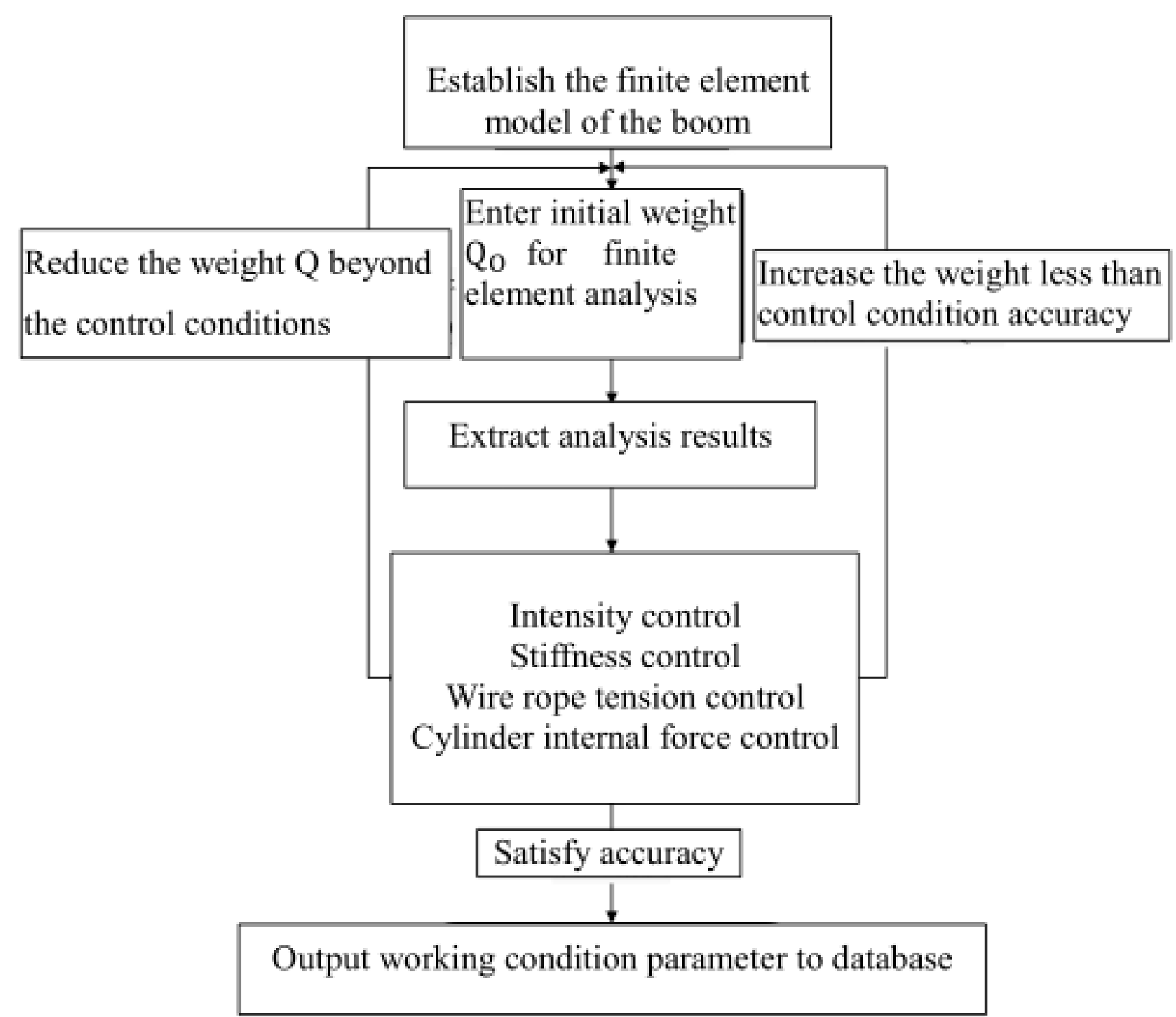

Figure 7. All-Terrain Crane Performance Calculation Process.

\subsubsection{Internal Force Controlling of Derricking Cylinder}

The most representative amplitude form of all-terrain crane telescopic boom finishing the luffing process is by hydraulic cylinder, as shown in figure 8. Hydraulic cylinder luffing mechanism has advantages of simple and compact construction, easy setting, large carrying capacity, smooth and reliable in operation, etc. According to the crane lifting capacity, double cylinder or single cylinder ball type valve can be adopted in luffing mechanism, the lower end of the variable amplitude oil cylinder rod is hinged on the rotary tables, and the upper end is hinged on reinforced hinge hole corresponding the middle part of the basic arm, it only bears axial force and doesn't bear bending moment in the work. According to the load characteristics of the hydraulic cylinder, the LINK10 unit is usually adopted in the finite element model to simulate the hydraulic cylinder. Hydraulic cylinder is a standard component, the specifications and features have been given before it is sold, it is ok to compare the maximum unit internal force $\mathrm{F} x$ of the hydraulic cylinder with the maximum load $[\mathrm{F}]$ in the hydraulic cylinder specifications by the method of extraction.

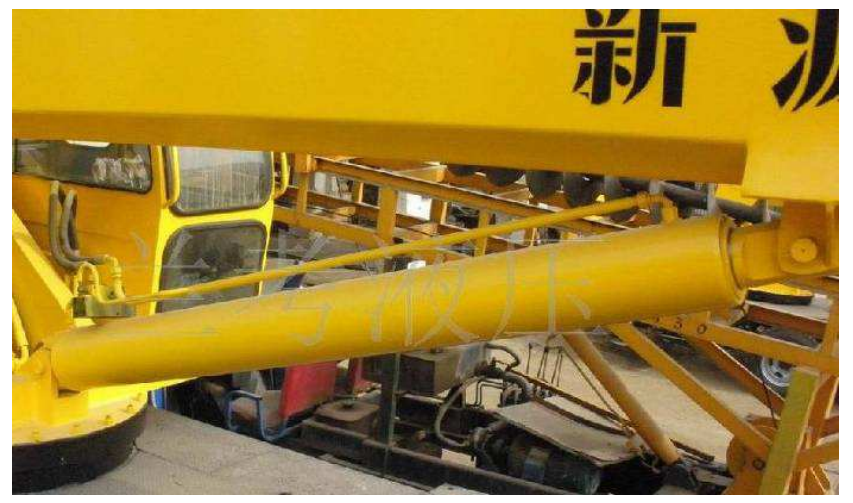

Figure 8. The luffing hydraulic cylinder. 


\subsubsection{Tension Control Conditions of Super Lifting Ropes}

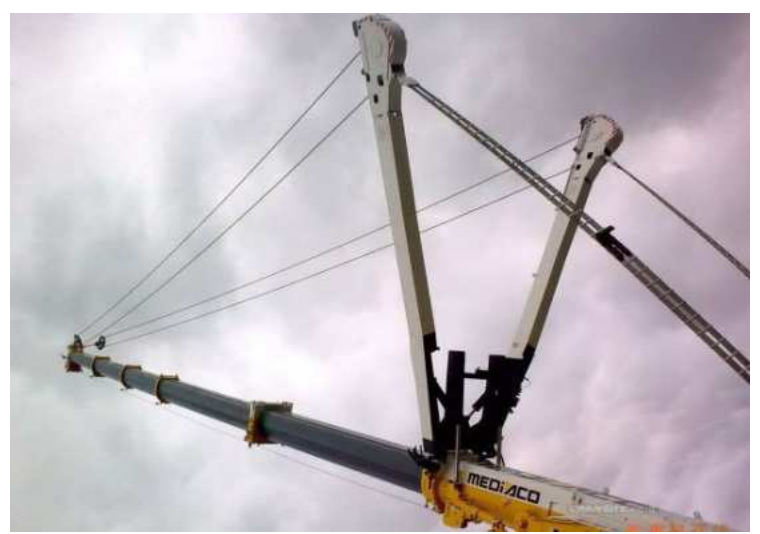

Figure 9. The Super structure.

In the super working condition of all-terrain cranes, the super steel wire rope and the super lifting plate are important flexible parts, which play an important role in the expansion of a crane's weight, as shown in figure 9. In the finite element analysis, the COMBIN39 element is used to simulate according to the characteristics of the super lifting rope. Based on the results by extraction, the wire rope tension $\mathrm{F}$ cannot exceed the specified load $[F]=F_{\max } / n$, where $F_{\max }$ is ultimate load of wire ropes, and $\mathrm{n}$ is the safety factor.

\subsection{Comparison of Performance Results}

According to actual lifting performance tables of fixed vice arm condition, which the enterprise given, 10 kinds of working conditions are analyzed and calculated in this paper, according to these controlling conditions such as the strength, stiffness, variable amplitude cylinder internal force, the performance results obtained are compared with the actual results, as shown in table 1.

Table 1. Comparison of lifting performance results.

\begin{tabular}{|c|c|c|c|c|c|c|c|c|}
\hline \multirow{2}{*}{$\begin{array}{l}\text { Working } \\
\text { Condition } \\
\end{array}$} & \multirow{2}{*}{$\begin{array}{l}\text { Main arm } \\
\text { Length(m) }\end{array}$} & \multirow{2}{*}{$\begin{array}{l}\text { Vice } \operatorname{arm} \\
\text { Length(m) }\end{array}$} & \multirow{2}{*}{$\begin{array}{l}\text { Vice-arm } \\
\text { Installation Angle }\left(^{\circ}\right)\end{array}$} & \multirow{2}{*}{$\begin{array}{l}\text { Range } \\
\text { (m) }\end{array}$} & \multicolumn{2}{|l|}{ Weight(t) } & \multirow{2}{*}{ Difference } & \multirow{2}{*}{$\begin{array}{l}\text { Error } \\
\text { Rate (\%) }\end{array}$} \\
\hline & & & & & Actual Value & Calculated Value & & \\
\hline 1 & 63.1 & 12.2 & 0 & 40 & 9.2 & 10.616 & 1.416 & 15.39 \\
\hline 2 & 73 & 12.2 & 40 & 50 & 7.2 & 6.923 & -0.277 & -5.09 \\
\hline 3 & 57.9 & 22 & 20 & 62 & 4.7 & 4.864 & 0.164 & 6.34 \\
\hline 4 & 57.9 & 22 & 20 & 62 & 5.7 & 5.870 & 0.170 & 5.41 \\
\hline 5 & 57.9 & 22 & 40 & 60 & 4.7 & 4.805 & 0.105 & 5.31 \\
\hline 6 & 57.9 & 22 & 40 & 60 & 5.7 & 5.808 & 0.108 & 4.52 \\
\hline 8 & 68 & 22 & 20 & 62 & 4.5 & 4.713 & 0.213 & 4.67 \\
\hline 9 & 68 & 22 & 20 & 62 & 5.5 & 5.737 & 0.237 & 4.18 \\
\hline 10 & 73 & 22 & 0 & 52 & 4.7 & 5.182 & 0.482 & 8.40 \\
\hline
\end{tabular}

Though comparing the analysis result with the actual lifting performance, we know that because the calculation of the lifting performance is only based on the calculation of arm frame structure, without considering the constraints that the lower part of the car affected the crane performance, so the calculation value of the lifting capacity is larger.

\subsection{Performance Tables Generation of All-Terrain Cranes}

After performance calculation of all-terrain cranes is completed, these data of the performance calculated by VBA technology and EXCEL program are outputted to the performance table template, methods for operating EXCEL object by using VBA language are similar to operation methods of the WORD object, method of data objects manipulated by EXCEL object is simpler and the output is more convenient. if VBA macro technology of EXCEL software is used, referencing the EXCEL dynamic link database by VB 6.0 , creating an application object in the EXCEL object library and opening the previously specified template file are needed, and performance parameters are outputted to the specified location, the specific operation is shown in figure 10 .

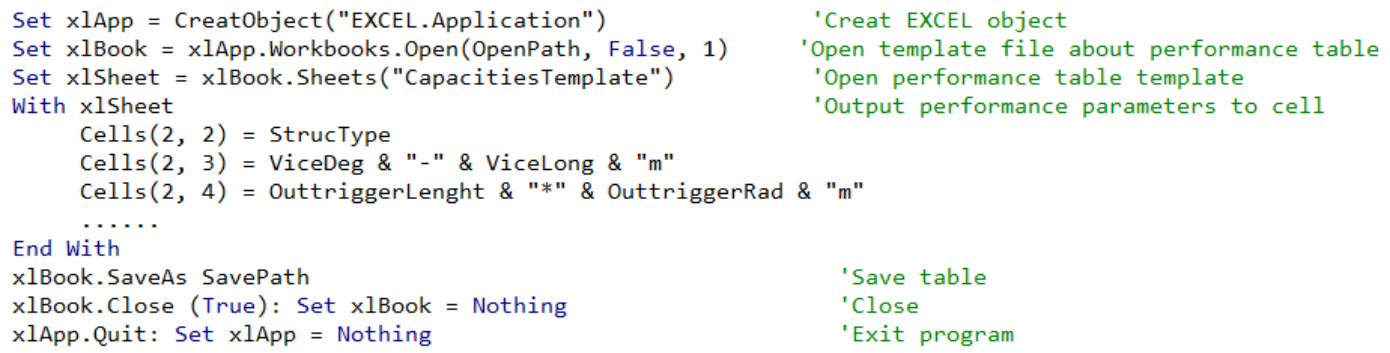

Figure 10. Codes of generating performance tables.

Considering the company's performance tables format as a template for the output in this paper, template is produced by using EXCEL form, the output effect is shown in figure 11. 


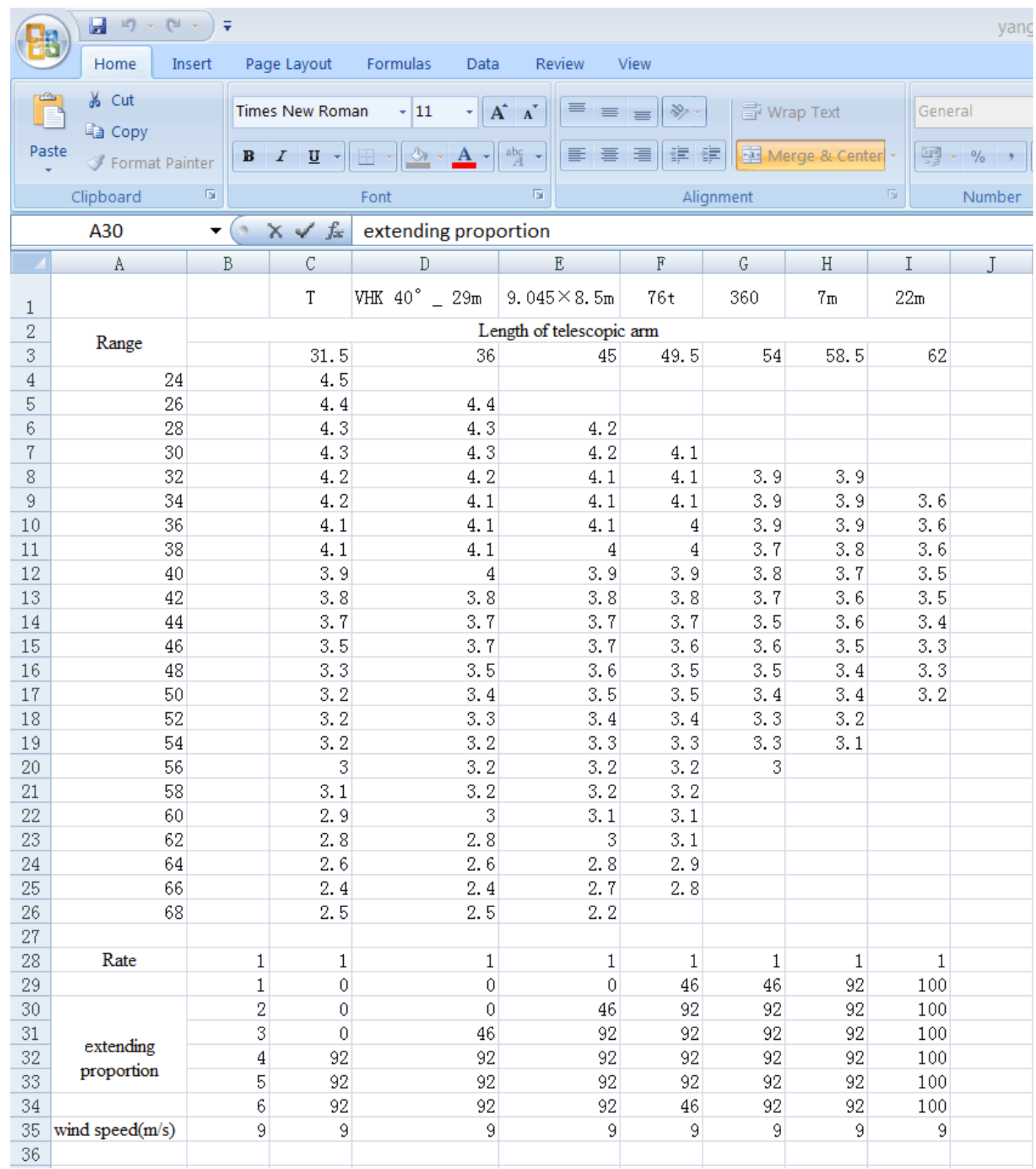

Figure 11. Output sample of performance tables.

\section{Conclusions}

In this paper, though VBA Macro Technology, finite element analysis and calculation on arm frame structure of all-terrain cranes are carried on, and automatic generation technology of working performance reports is also realized, class encapsulation of VBA macro statement by using VB6.0 is introduced. VBA macro statements are encapsulated, which reduces greatly the difficulty of secondary development of the Word and Excel procedures, outputting efficiently calculation reports and performance tables are achieved, which provides a convenience for rapid development and design of new products.

\section{References}

[1] Dong J, Cheng W M, Ren Y Z, et al. Research on the Structural Performance of Large Tonnage Gantry encapsulated [J]. Advanced Materials Research, 2013, 823: 247-250.

[2] Sandip D. Shinde. Standardization of Vice arm Crane Design by "F. E. M. Rules" And Parametric Modeling [J]. International Journal of Recent Trends in Engineering, 2009,5: 145-149.

[3] Maedo, Teppei;Ichikawa, Yasuo. Weight and width reductions of Latticed Boom Crawler Cranes [J]. Research and Development Kobe Steel Engineering Reports, 2012,8 (62): 41-44. 
[4] Dušan Kovačević, Slobodan Ranković. Fem Modeling Of Spatial Structural Systems In Evaluation Of The Real Structural Performances [J]. Architecture and Civil Engineering, 2011, 9: 347-356.

[5] Zhang D, Cheng Q, Agterberg F, et al. An improved solution of local window parameters setting for local singularity analysis based on Excel VBA batch processing technology[J]. Computers \& Geosciences, 2016, 88: 54-66.

[6] Cox B J. Object oriented programming. an evolutionary approach $[\mathrm{J}]$. Proceedings of the Usenix C++ Workshop Santa Fe Nm, 1986, 14 (2): 350-360.

[7] Liu Y P. Automatic Producing Examination Paper Analysis with the Help of VBA Technology [J]. Journal of Wuhan Institute of Shipbuilding Technology, 2004.
[8] Harrison D R, Mabry Iii C B, Beer W A, et al. Frame structure for lift crane machinery: US5598935[P]. 1997.

[9] Zhang J, Xu W, Li J, et al. Research on loaded rule of boom of all-terrain crane [J]. Hoisting \& Conveying Machinery, 2014.

[10] Payette G S, Reddy J N. A Nonlinear Finite Element Framework for Viscoelastic Beams Based on The High-Order Reddy Beam Theory [J]. Journal of Engineering Materials and Technology, 2013, 1 (135): 1-11.

[11] Liu Y, Qin Y, Zhang Q, et al. Non-linear analysis and convergence research on telescopic boom of all-terrain crane [J]. Hoisting \& Conveying Machinery, 2014.

[12] Yao J, Qiu X, Zhou Z, et al. Buckling Failure Analysis of All-terrain Crane Telescopic Boom Section [J]. Engineering Failure Analysis, 2015, 57: 105-117. 